\title{
Modification of the eikonal relation for chemical waves to include fluid flow
}

\author{
J. W. Wilder \\ Department of Mathematics, West Virginia University, Morgantown, West Virginia 26506 \\ D. A. Vasquez and B. F. Edwards \\ Department of Physics, West Virginia University, Morgantown, West Virginia 26506
}

(Received 26 August 1992)

\begin{abstract}
Propagating wave fronts resulting from autocatalytic chemical reactions have been the focus of much recent research. For the most part, the hydrodynamics resulting from such reactions has been neglected. In this work, a relation is derived for the normal speed of a propagating wave front as a function of the local curvature when fluid motion is allowed. This "eikonal" equation is a generalization of one which was derived in the absence of fluid flow. It is also shown that small variations in the fluid density due to the chemical reaction do not change the form of the relation.
\end{abstract}

PACS number(s): 03.40.Gc, 47.15. $-\mathrm{x}, 47.20 . \mathrm{Bp}, 47.70 . \mathrm{Fw}$

\section{INTRODUCTION}

Traveling waves resulting from chemical reactions have been observed in several chemical systems [1-6] such as the Belousov-Zhabotinskii (BZ) and iodatearsenous-acid reactions. An important class of these traveling fronts which has been of interest is the evolution of spiral waves. The propagation of waves in twodimensional excitable media has been studied by several researchers. In particular, Tyson and Keener [7-9] derived a relation for the normal velocity of the wave front using singular perturbation theory. They applied this approach to the case of the Belousov-Zhabotinskii reaction. In analyzing this system, the effects of fluid motion were neglected. The result was an eikonal equation which explicitly shows the dependence of the normal velocity of the wave front on the curvature of the front [7-9].

Recent theoretical work on the iodate-arsenous-acid system [10-12] has shown that convective motion of the fluid plays an important role in the propagation of the front in this system. This importance is due to a small density difference between the reacted and unreacted fluids. This difference leads to buoyancy-driven convection, which is believed to result in the experimental observation of curved propagation fronts in cylinders when the reaction is initiated at the bottom of a vertical tube $[13,14]$. Also observed is an enhancement of the front velocity: the velocity is greater under conditions such that convection can take place. In attempting to model this behavior, it is necessary to study the influence of fluid motion on the system, since reaction-diffusion theory alone cannot account for these observed characteristics. Recent work has used a modified relation for the normal velocity of the front which includes the effect of the underlying fluid motion. It is the purpose of this work to derive this relation in a more formal manner in order to justify its use.

In deriving the modified eikonal relation we shall use the same model for the $\mathrm{BZ}$ reaction as that used by Keener and Tyson [7]:

$$
\begin{aligned}
& A+R \underset{k_{1}}{\longrightarrow} U+P, \\
& U+R \underset{k_{2}}{\longrightarrow} 2 P, \\
& A+U \underset{k_{3}}{\longrightarrow} 2 U+2 V, \\
& 2 U \underset{k_{4}}{\longrightarrow} A+P, \\
& B+V \underset{k_{5}}{\longrightarrow} h R,
\end{aligned}
$$

where $A=\left[\mathrm{BrO}_{3}{ }^{-}\right], B=[$ bromomalonic acid] $+[$ malonic acid $], \quad P=[\mathrm{HOBr}], \quad U=\left[\mathrm{HBrO}_{2}\right], \quad V=\left[\mathrm{Ce}^{4+}\right]$, $R=\left[\mathrm{Br}^{-}\right]$, the $k_{i}$ 's are rate constants, and $h$ is a stoichiometric parameter. With the assumptions that $A$ and $B$ remain constant, and that $R$ is in quasiequilibrium, the system is reduced to involving only two variables, $U$ and $V$. One can now write the mass action kinetic equations for these variables, and include diffusive and hydrodynamic effects. We shall first consider a case where the fluid density is constant in both space and time, and then consider one in which the density varies as a result of the chemical reaction.

\section{CONSTANT FLUID DENSITY}

After nondimensionalization, one arrives at a system of partial differential equations for the above model of the form

$$
\begin{aligned}
& \epsilon u_{t}+\epsilon \mathbf{w} \cdot \nabla u=\epsilon^{2} \nabla^{2} u+u-u^{2}-\hat{f} v \frac{u-q}{u+q}, \\
& v_{t}+\mathbf{w} \cdot \nabla v=\epsilon \nabla^{2} v+u-v, \\
& \mathbf{w}_{t}+(\mathbf{w} \cdot \nabla) \mathbf{w}=-\nabla p_{r}+\lambda \nabla^{2} \mathbf{w}, \\
& \boldsymbol{\nabla} \cdot \mathbf{w}=0,
\end{aligned}
$$

where $\quad u=\left(2 k_{4} / k_{3} A\right) U ; \quad v=\left[k_{4} k_{5} B /\left(k_{3} A\right)^{2}\right] V$; $\mathbf{w}=\left(k_{3} A D\right)^{-1 / 2} \times$ (fluid velocity); $x=\left(k_{5} B / \sqrt{k_{3} A D}\right)$ $\times($ space $) ; \quad t=k_{5} B \times($ time $) ; \quad p_{r}=\rho k_{3} A D \times($ reduced 
pressure); $\hat{f}=2 h, q=2 k_{1} k_{4} / k_{2} k_{3} ; \epsilon=k_{5} B / k_{3} A$ (which is 0.01 using the parameters of Tyson and Keener and therefore assumed small) with $D$ being the diffusion coefficient for the reacting species; $\rho$ is the density of the fluid, and $\lambda$ is the nondimensional viscosity of the fluid given by $\lambda=v k_{5} B / \rho\left(k_{3} A D\right)^{3 / 2}$, which would have a value of 6.07 for water with the above model. Note that the pressure is written as a reduced pressure meaning that we have used $\boldsymbol{\nabla}$ (reduced pressure) $=\boldsymbol{\nabla}$ (pressure) + const $\times g \hat{\mathbf{g}}$, where $g$ is the acceleration of gravity, and $\hat{\mathbf{g}}$ is the direction opposite to that in which gravity acts. For simplicity, we shall assume that the direction of propagation of the front and $\hat{\mathbf{g}}$ are aligned (i.e., $\hat{\mathbf{g}}=\widehat{\mathbf{x}}$ ).

\section{A. One-dimensional waves}

It is useful to first consider the case of one-dimensional waves. This case allows one to solve the problem with little effort, and shows the basic ideas behind the singular perturbation theory. Following the method of Keener and Tyson [7] we can define two regions: one in which the variables are slowly varying spatially, and one in which they vary rapidly. In the slowly varying region we have [setting $\epsilon=0$ in (2)]

$$
\begin{aligned}
& f(u, v)=u-u^{2}-\hat{f} v \frac{u-q}{u+q}=0, \\
& v_{t}+w \frac{\partial v}{\partial x}=g(u, v)=u-v, \\
& \frac{\partial w}{\partial x}=0, \\
& \frac{\partial w}{\partial t}=-\frac{\partial p}{\partial x},
\end{aligned}
$$

where $w$ is the fluid velocity. For the $\mathbf{B Z}$ reaction, there are three solutions of (3a). Since one of these is unstable, there are two solutions denoted by $u=U_{ \pm}(v)$ which are valid far away from the front. Substitution of these solutions into (3b) allows for the solution of the remaining equations. The resulting solution for $v$ will be referred to as the outer solution. Discontinuities in $u$ which are permitted by this outer solution for $v$ must be patched up by using a moving boundary layer of the form $\xi=(x-c t) / \epsilon$, with $u=u(\xi)$. This leads to equations in the boundary layer of the form

$$
\begin{aligned}
& u_{\xi \xi}+(c-w) u_{\xi}+f(u, v)=0, \\
& v_{\xi \xi}+(c-w) v_{\xi}+\epsilon g(u, v)=0, \\
& w_{\xi}=0 .
\end{aligned}
$$

As $\xi \rightarrow \pm \infty$, the solution of (4) must match the outer solution discussed above. If we examine (4b), set $\epsilon=0$, and require $v$ to remain bounded as $\xi \rightarrow \pm \infty$, it is easily seen that $v(\xi)=v_{0} \equiv$ const, $w(\xi)=w_{0} \equiv$ const. Equation (4a) then represents an eigenvalue problem for the speed of propagation $c$ as a function of $v_{0}$ and $w_{0}$, the fixed values of $v$ and $w$ in the boundary layer. If we let $d=c-w_{0}$ we arrive at exactly the equation derived by Keener and Tyson. Thus, for any given fluid velocity $w_{0}$, it is easily seen that the speed of the front in the presence of fluid flow can be related to that without the fluid flow by

$$
c_{\text {with }}=c_{\text {without }}+w_{0},
$$

as one would have expected in this simple case.

\section{B. Two-dimensional waves}

This case can also be examined by considering the slowly varying region and a thin boundary layer. To accommodate nonplanar fronts a change of variables is made to a coordinate system moving with the boundary layer such that $x=X(\epsilon \xi, \eta, \tau), y=Y(\epsilon \xi, \eta, \tau), t=\tau$. As discussed by Keener and Tyson, we now seek solutions for which the $\eta$ coordinate gives level surfaces of $u$, with wave front motion entirely depicted by movement of the coordinate system. Thus, we want solutions such that $u=u(\xi)$ with $\xi=0$ locating the wave front. Without loss of generality, the $\xi$ coordinate can be scaled such that $X_{1}^{2}+Y_{1}^{2}=1$, where the subscript 1 denotes partial differentiation with respect to the first argument. Also note that in this traveling coordinate system, $u$ is independent of $\eta$ and $\tau$. This transformation leads to much more complicated equations in the boundary layer than the one-dimensional case, and so we shall only write down the leading-order terms in $\epsilon$ since only these are needed for the analysis to be done here. The leadingorder terms are of the form

$u_{\xi \xi}+\left[(N-\epsilon K)-\frac{\left(w_{1} Y_{\eta}-w_{2} X_{\eta}\right)}{\left(X_{\eta}^{2}+Y_{\eta}^{2}\right)^{1 / 2}}\right] u_{\xi}+f(u, v)=0$,

$v_{\xi \xi}+\left[(N-\epsilon K)-\frac{\left(w_{1} Y_{\eta}-w_{2} X_{\eta}\right)}{\left(X_{\eta}^{2}+Y_{\eta}^{2}\right)^{1 / 2}}\right] v_{\xi}+O(\epsilon)=0$,

$\mathbf{w}_{\xi \xi}+\boldsymbol{O}(\epsilon)=\mathbf{0}$,

where $w_{1}$ and $w_{2}$ are the components of the fluid velocity, and $N$ and $K$ are the normal velocity of the front and its curvature, respectively, and are given by

$$
\begin{aligned}
& N=\frac{X_{\tau} Y_{\eta}-Y_{\tau} X_{\eta}}{\left(X_{\eta}^{2}+Y_{\eta}^{2}\right)^{1 / 2}}, \\
& K=\frac{Y_{\eta} X_{\eta \eta}-X_{\eta} Y_{\eta \eta}}{\left(X_{\eta}^{2}+Y_{\eta}^{2}\right)^{3 / 2}} .
\end{aligned}
$$

If $\mathbf{w}$ is to remain bounded as $\xi \rightarrow \pm \infty$, then (5c) implies that both components of the fluid velocity are independent of the normal coordinate $\xi$. Even though $N$ and $K$ depend on $\xi$ in general, it has been shown that [8]

$$
N-\epsilon K=\left.(N-\epsilon K)\right|_{\xi=0}+O\left(\epsilon^{2}\right) \text {. }
$$

Similar arguments lead to the conclusion that

$$
\frac{\left(w_{1} Y_{\eta}-w_{2} Y_{\eta}\right)}{\left(X_{\eta}^{2}+Y_{\eta}^{2}\right)^{1 / 2}}=\left[\frac{\left(w_{1} Y_{\eta}-w_{2} X_{\eta}\right)}{\left(X_{\eta}^{2}+Y_{\eta}^{2}\right)^{1 / 2}}\right]_{\xi=0}+O(\epsilon) .
$$

Thus, to order $\epsilon$ we can consider the coefficients of $u_{\xi}$ 
and $v_{\xi}$ in Eqs. (5a) and (5b) to be independent of $\xi$. Using this along with $(5 \mathrm{~b})$ leads to the conclusion that $v$ is not independent of $\xi$ to order $\epsilon$. Thus, we have

$$
\begin{aligned}
& \mathbf{w}=\mathbf{w}_{0}(\eta, \tau), \\
& v=v_{0}(\eta, \tau) .
\end{aligned}
$$

Since we are interested in how the velocity of the front is affected by the curvature of the front as well as the fluid flow, let us compare (5a) to the case when there are no curvature effects or fluid flow, i.e., (4a) with $w=0$. This results in the relation

$$
\left[N-\epsilon K-\frac{\left(w_{1} Y_{\eta}-w_{2} X_{\eta}\right)}{\left(X_{\eta}^{2}+Y_{\eta}^{2}\right)^{1 / 2}}\right]_{\xi=0}=c\left(v_{0}\right)+\boldsymbol{O}(\epsilon) .
$$

Recognizing that the third term on the left-hand side is the component of the fluid velocity in the direction of the normal to the wave front, and ignoring terms of order $\epsilon$, we arrive at a condition for the wave front given by

$$
N=c\left(v_{0}\right)+\hat{\mathbf{n}} \cdot \mathbf{w}_{0}+\epsilon K,
$$

where $\hat{\mathbf{n}}$ is the normal to the wave front and $c\left(v_{0}\right)$ represents the velocity of the front in the absence of fluid motion and curvature effects. As done by Keener and Tyson, we retain the term involving the curvature since it may become appreciable in some applications.

\section{NONCONSTANT FLUID DENSITY}

Recent theoretical work on the iodate-arsenous-acid system has shown that in that problem convection is driven by a small density difference between the reacted and unreacted fluids [10-12]. Since similar considerations may be necessary for other systems as well if one wishes to study the hydrodynamic stability of the fronts produced by these chemical reactions, we shall now consider a fluid which undergoes a change in density as the reaction takes place. When we allow for a varying fluid density, the fluid equations in dimensioned form become

$$
\begin{aligned}
& \frac{\partial(\rho \mathbf{W})}{\partial T}+(\mathbf{W} \cdot \nabla)(\rho \mathbf{W})=-\rho g \hat{\mathbf{x}}-\nabla P+\nu \nabla^{2} \mathbf{W}, \\
& \frac{\partial \rho}{\partial T}+\nabla \cdot(\rho \mathbf{W})=0,
\end{aligned}
$$

where $\mathrm{W}$ is the fluid velocity, $P$ the pressure, $v$ the viscosity of the fluid, $g$ the acceleration of gravity, and $T$ the dimensioned time. In this problem we are thinking of the change in density as a result of the chemical reaction. Since the total mass of the solution remains constant, the density change must be a result of a volume change due to the reaction. Thus, we can write

$$
V(U)=V_{0}+\left(U_{0}-U\right) \delta V_{u},
$$

where $V(U)$ is the volume of the solution with concentration $U$ resulting from a reaction where the solution initially had a concentration $U_{0}$ and volume $V_{0}$, and where $\delta V_{u}$ represents the molar volume change due to the reaction. Using this we can write the density as

$$
\rho=\frac{M}{V_{0}+\left(U_{0}-U\right) \delta V_{u}}=\frac{M}{V_{0}}\left[\frac{1}{1+\frac{\delta V_{u}}{V_{0}}\left(U_{0}-U\right)}\right]
$$

where $M$ is the mass. Since $\left(\delta V_{u} / V_{0}\right)\left(U_{0}-U\right)$ is a small quantity (for the iodate-arsenous-acid system, for example, the relative density change is on the order of $10^{-4}$ ), we can expand this as

$$
\begin{aligned}
\rho & \simeq \frac{M}{V_{0}}\left[1-\frac{\delta V_{u}}{V_{0}}\left(U_{0}-U\right)\right] \\
& \simeq \rho_{0}\left[1-\frac{\delta V_{u}}{V_{0}}\left(U_{0}-U\right)\right] .
\end{aligned}
$$

Since we are considering the case of a front moving vertically, with the concentration being $U_{+}$and the fluid density $\rho_{+}$far in front of the traveling wave, we can let this be our reference state yielding

$$
\rho \simeq \rho_{+}\left[1-\frac{\delta V_{u}}{V_{0}}\left(U_{+}-U\right)\right],
$$

where $\rho_{+}$and $U_{+}$are constants in space and time. Using this in (12) and nondimensionalizing in a manner analogous to that used above yields

$$
\begin{aligned}
& \mathbf{w}_{t}+(\mathbf{w} \cdot \boldsymbol{\nabla}) \mathbf{w}+\widetilde{\epsilon}\left[\frac{\partial(u \mathbf{w})}{\partial t}+(\mathbf{w} \cdot \nabla)(u \mathbf{w})\right] \\
& =-\nabla p_{r}+\lambda \nabla^{2} \mathbf{w},-\widetilde{\epsilon} G u \widehat{\mathbf{x}}, \\
& \boldsymbol{\nabla} \cdot \mathbf{w}+\widetilde{\epsilon}\left[\frac{\partial(u)}{\partial t}+\nabla \cdot(u \mathbf{w})\right]=0,
\end{aligned}
$$

where $\quad \widetilde{\epsilon}=\delta V_{u} k_{3} A / 2 V_{0} k_{4}=(0.0012) \delta V_{u} / V_{0}<\epsilon, \quad G$ $=g / k_{5} B \sqrt{k_{3} A D}$. The second equality for $\widetilde{\epsilon}$ is a result of using the parameters given in the work of Keener and Tyson [7]. The nondimensionalization is the same as that used above except $\rho$ is replaced by $\rho_{+}$.

Since $\widetilde{\epsilon}<\epsilon$, to first order in $\epsilon$ we can neglect the terms in (17) which are on the order of $\widetilde{\epsilon}$, resulting in

$$
\begin{aligned}
& \mathbf{w}_{t}+(\mathbf{w} \cdot \boldsymbol{\nabla}) \mathbf{w}=-\nabla p_{r}+\lambda \nabla^{2} \mathbf{w}-\widetilde{\epsilon} G u \hat{\mathbf{x}}, \\
& \boldsymbol{\nabla} \cdot \mathbf{w}=0,
\end{aligned}
$$

where we have kept the last term on the right-hand side of (18a) because of the largeness of $G$ which causes $\widetilde{\epsilon} G$ not to be small (using the parameter values for this model we find $G=2.1 \times 10^{6}$ ). The equations involving the concentrations of the species are not changed when we consider a varying density, and so we have not restated them. If we now transform the equations as was done above, we find that in the boundary layer, the variables satisfy

$u_{\xi \xi}+\left[(N-\epsilon K)-\frac{\left(w_{1} Y_{\eta}-w_{2} X_{\eta}\right)}{\left(X_{\eta}^{2}+Y_{\eta}^{2}\right)^{1 / 2}}\right] u_{\xi}+f(u, v)=0$, 
$v_{\xi \xi}+\left[(N-\epsilon K)-\frac{\left(w_{1} Y_{\eta}-w_{2} X_{\eta}\right)}{\left(X_{\eta}^{2}+Y_{\eta}^{2}\right)^{1 / 2}}\right] v_{\xi}+O(\epsilon)=0$,

$\lambda \mathbf{w}_{\xi \xi}-\widetilde{\epsilon} \epsilon^{2} G u \hat{\mathbf{x}} \cdot \hat{\mathbf{k}}+O(\epsilon)=0$,

$Y_{\eta} \frac{\partial w_{1}}{\partial \xi}-X_{\eta} \frac{\partial w_{2}}{\partial \xi}+O(\epsilon)=0$,

where $\hat{\mathbf{k}}=\hat{\boldsymbol{\xi}}$ when the $\xi$ component of (19c) is considered, and is $\hat{\eta}$ when the $\eta$ component is considered. Equation (19d) is the transform of (18b) which was not stated in the previous section since it was not needed [since $(5 \mathrm{c})$ led to the conclusion that both components of the fluid velocity were constants in $\xi]$. Note that $\tilde{\epsilon} \epsilon^{2} G \sim 1$; if we expect a relative density difference on the order of $10^{-4}$ (as it is for such systems as the iodate-arsenous-acid reaction), with a change in concentration on the order of $5 \times 10^{-2} \mathrm{M}$ [14], (16) predicts $\delta V_{u} / V_{0}=2$. Using this gives $\widetilde{\epsilon} \epsilon^{2} G=0.5$. While these values would change slightly with the experimental conditions considered, we can see that this quantity will roughly be $\sim 1$. Thus, we can no longer conclude that the fluid density is a constant in $\xi$ as it was in the previous section.

While for the previous case the fluid velocity was independent of $\xi$ to first order in $\epsilon$, this is no longer true. Thus, we must look more closely at the behavior of the coefficient of $u_{\xi}$ in (19a). We already know that $N-\epsilon K$ is independent of $\xi$ to second order in $\epsilon$, and so all we need to show is that the third term in this coefficient is independent of $\xi$ at least to first order in $\epsilon$ and then we will be able to draw the association between the coefficient of $u_{\xi}$ in (19a) and the speed of the front as was done previously. If we expand this third term in a power series in $\xi$ about $\xi=0$ we find

$$
\begin{aligned}
\frac{\left(w_{1} Y_{\eta}-w_{2} X_{\eta}\right)}{\left(X_{\eta}^{2}+Y_{\eta}^{2}\right)^{1 / 2}}= & {\left[\frac{\left(w_{1} Y_{\eta}-w_{2} X_{\eta}\right)}{\left(X_{\eta}^{2}+Y_{\eta}^{2}\right)^{1 / 2}}\right]_{\xi=0} } \\
+ & {\left[\left[\frac{\left(w_{1}^{\prime} Y_{\eta}-w_{2}^{\prime} X_{\eta}\right)}{\left(X_{\eta}^{2}+Y_{\eta}^{2}\right)^{1 / 2}}\right]_{\xi=0}\right.} \\
& \left.+\epsilon\left[\frac{\left(w_{1} Y_{\eta}^{\prime}-w_{2} X_{\eta}^{\prime}\right)}{\left(X_{\eta}^{2}+Y_{\eta}^{2}\right)^{1 / 2}}\right]_{\xi=0}\right] \xi \\
& +O\left(\xi^{2}\right),
\end{aligned}
$$

where the prime denotes differentiation with respect to $\xi$. If we examine the second term on the right-hand side of (20), we see that it is zero due to (19d). This results in all of the terms on the right-hand side of (20) other than the first being $O(\epsilon)$. Thus, even though the fluid velocity is now a function of $\xi$, the coefficient of $u_{\xi}$ in (19a) is still independent of $\xi$ to first order in $\epsilon$, and we arrive at the same relation for the normal speed of the front as was found in the previous section, namely the relation given in (11).

\section{CONCLUSIONS}

Using singular perturbation theory, an eikonal equation has been derived for the case of a propagating wave front in the presence of fluid motion. This represents a generalization of the relation which had previously been derived which neglected the effects of fluid motion. The modification of the relation made necessary by the presence of fluid flow is of the form expected, namely the speed of the front is modified by the addition of the normal component of the fluid velocity at the front, as was expected. The use of this modified form in previous work based on the assumption that this would be the form of the new equation is thus justified. It has also been shown that the form of this relation is not altered by allowing for small density changes due to the chemical reaction. Recent research on the iodate-arsenous-acid system made these calculations necessary in that they show that hydrodynamic effects must be considered in order to adequately explain the experimentally observed phenomena. While the work presented here has used the $\mathrm{BZ}$ reaction as a prototype, the results are applicable to other systems. For example, if one is interested in the iodate-arsenousacid system, one merely needs to change the form of $f(u, v)$ and $g(u, v)$ as well as use the appropriate rate constants to obtain the analogous front equation for this reaction. Now that the relation involving fluid motion has been derived, it shall be used in further studies concerned with the propagation of reaction-diffusion fronts where fluid flow is important.

\section{ACKNOWLEDGMENTS}

This work was supported in part by NSF Grant No. RII-8922106, and by a grant from the National Research Center for Coal and Energy.
[1] G. Bazsa and I. R. Epstein, J. Phys. Chem. 89, 3050 (1985).

[2] R. J. Field and R. M. Noyes, J. Am. Chem. Soc. 96, 2001 (1974)

[3] R. J. Gowland and G. Stedman, J. Chem. Soc. Chem. Commun. 1983, 1038 (1983).

[4] A. Hanna, A. Saul, and K. Showalter, J. Am. Chem. Soc. 104, 3838 (1982).

[5] A. Saul and K. Showalter, in Oscillations and Traveling Waves in Chemical Systems, edited by R. J. Field and M. Burger (Wiley, New York, 1985), pp. 419-439.

[6] D. M. Weitz and I. R. Epstein, J. Phys. Chem. 88, 5300 (1984).
[7] J. P. Keener and J. J. Tyson, Physica 21D, 307 (1986).

[8] J. P. Keener, SIAM J. Appl. Math. 46, 1039 (1986).

[9] J. J. Tyson and J. P. Keener, Physica D 32, 327 (1988).

[10] B. F. Edwards, J. W. Wilder, and K. Showalter, Phys. Rev. A 43, 749 (1991).

[11] J. W. Wilder, B. F. Edwards, and D. A. Vasquez, Phys. Rev. A 45, 2320 (1992).

[12] D. A. Vasquez, B. F. Edwards, and J. W. Wilder, Phys. Rev. A 43, 6694 (1991).

[13] T. McManus, Ph.D. thesis, West Virginia University, 1989 (unpublished).

[14] J. A. Pojman, I. R. Epstein, T. J. McManus, and K. Showalter, J. Phys. Chem. 95, 1299 (1991). 